\title{
Molecular identification of a native wine yeast from bubod used in tapuy fermentation
}

\author{
Claire A. Ablang ${ }^{1}$, Kyle Noreen C. Alvar ${ }^{1}$, Kyle Aaron T. Samson ${ }^{1}$, E Librado A. Santiago ${ }^{1-3 *}$ \\ ${ }^{1}$ Department of Biochemistry, Faculty of Pharmacy; ${ }^{2}$ Research Center for the Natural and Applied \\ Sciences; ${ }^{3}$ Graduate School, University of Santo Tomas, 1015 Manila, Philippines
}

\begin{abstract}
A popular commercial traditional Filipino rice wine, locally known as tapuy from Banaue and Mountain Province, is made from a natural starter culture called bubod. Bubod is a white, oval, and disc-shaped hardened starch powder that contains a mixed cultures of molds, several yeasts and lactobacilli bacteria which carry out the saccharification and natural fermentation process. The study aimed to isolate, characterize, purify the DNA of the principal yeast in bubod and establish its molecular identity using PCR amplification and DNA sequencing. The native wine yeast was initially propagated on peptone yeast glucose (PYG) agar and broth, isolated and purified by pour plate and streak plate method. The yeast isolate reproduced by unilateral budding and fermented only glucose. Molecular identification of the isolated yeast from Bubod was Pichia kudriavzevii at 78\% homology. The morphological characteristics, unilateral budding and ability to ferment solely glucose matched and confirmed the yeast identity.
\end{abstract}

Keywords: bubod, tapuy, rice wine, DNA barcoding, Saccharomyces, Pichia kudriavzevii

\section{INTRODUCTION}

The main ingredients in the commercial preparation of traditional Filipino rice wine locally called tapuy consist of waxy glutinous milled rice, often pretoasted, and bubod [1]. Bubod is a natural powdered rice starter culture that gives quality to tapuy for its exquisite taste and aroma compounds. It contains a variety of microbial load ranging from molds (e.g., Rhizopus oryzae, Mucor sp.), yeasts (e.g., Sacchromyces cerevisiae), and bacteria (e.g., Lactobacillus sp.) which may influence to a certain extent the fermentation efficiency,

*To whom correspondence should be addressed: santiagolibrado@yahoo.com alcohol yield and flavor [2]. Regarded as man's oldest industrially friendly unicellular organisms, yeasts are widely used in the preparation of alcoholic beverages, bread, and a large variety of industrial products. They are evolutionally diverse and are known to typically grow asexually by budding [3].

Yeasts are generally recognized as safe (GRAS) [4]. Yeasts serve as the vibrant component in the fermentation process that convert sugar into alcohol, an ingredient shared in beer, wine and distilled beverages. Various yeast strains have differing physiological and fermentative properties, hence, the actual strain of yeast selected can have a direct influence on the finished wine [5]. 
Tapuy is a traditional rice wine exclusively produced in the Philippines particularly in Banaue and the Mountain Province. It is best served in important occasions such as weddings, rice harvesting ceremonies, fiestas, and cultural fairs [6]. Tapuy is produced from either pure glutinous rice or a combination of glutinous and non-glutinous rice together with onuad roots, ginger extract, and bubod.

Tapuy is a clear full-bodied wine with a strong alcoholic flavor and alcohol content of 28 proof equivalent to approximately $14 \% \mathrm{v} / \mathrm{v}$. It has no sulfites which are regular preservatives in other wines that sometimes cause adverse reactions like hang-over and allergies [7]. Unlike other alcoholic beverages, tapuy is not diluted with water and has no added sugar. It is consumed normally after one month of fermentation without further processing [8]. PhilRice is now into commercial manufacture of tapuy.

Rice wine production is a profitable venture in the Philippines. The tapuy is commercially available in the country's major wine stores and only a small percentage is allotted for export [9].

According to the observations of ChinteSanchez (2008), the microbial load of bubod differs, the number of yeasts and bacteria varies significantly from one locality to another [8]. Different strains of Saccharomyces, Aspergillus, and Lactobacillus were isolated. The fermentation efficiency of the different bubod also ranges from $6.55 \%$ to $15.04 \%$. This was due to the different count of natural saccharifying and alcohol-fermenting microorganisms in it [9]. Hence, bubod becomes an important determinant of good fermentation efficiency in tapuy.

Thus, evaluation and selection of yeast strains from bubod are required to further increase yield and alcohol content of tapuy. At the current state, its manufacture still needs improvement in its overall production.
Henceforth, the process of purifying wine sources as a single, and proven strain of yeasts has become the objective of this study; that is to isolate, and purify the native wine yeast from bubod. Secondly, to extract, purify and sequence its DNA in order to identify the yeast strain.

\section{EXPERIMENTAL}

Materials and reagents. The native wine yeast sample was isolated from bubod which was purchased from Mines View Park, Baguio City. Yeast extract powder at $0.125 \%$, dextrose powder at $0.3 \%$, peptone at $0.125 \%$ in $500 \mathrm{~mL} \mathrm{H}_{2} \mathrm{O}$ were purchased from Himedia. Glucose, fructose, and sucrose were manufactured by Merck. Lysis buffer and tris-EDTA (TE) buffer were prepared using analytical grade chemicals.

Isolation of yeast. ISP medium peptone yeast glucose (PYG) agar medium (from Himedia Laboratories) was used for plating. Cultures of yeasts were made by pour plating or streaking the yeast sample from the PYG broth inoculum onto the prepared agar plates. The Petri dishes were incubated upside down at $30^{\circ} \mathrm{C}$ for 24 $48 \mathrm{~h}$ and then the colonies were examined and counted. Dominant colonies were picked up and transferred to PYG agar slant.

Purification of yeast. Single colonies of representative isolate were purified following the dilution plating technique in PYG agar medium. Separated colonies were transferred again to PYG agar slants. Purification was done by streaking on plated agar and repeated twice or until pure cultures were obtained, as confirmed by microscopic examination.

Morphological characterization. The cellular morphology of the cultured yeasts was analyzed and viewed under a phase contrast microscope. Yeast sample was supplemented in a drop of sterile distilled water and placed on glass slide and then smeared until it dries off. The smear was stained using diluted methylene blue dye, 
air dried and observed under light microscope at $10 \times, 40 \times$, and $100 \times$ magnification. Budding and elevation of the cells in PYG slants were observed.

Carbohydrate fermentation test. Peptone at $1 \mathrm{~g}$, sodium chloride at $0.5 \mathrm{~g}$, phenol red at $0.0189 \mathrm{mg}$ and sugars at $2 \mathrm{~g}$ with glucose, sucrose, and fructose were weighed and dissolved in $100 \mathrm{~mL}$ distilled water. This served as the basal media. The miniaturized fermentation test was carried out in a 9-MicroWell ${ }^{\mathrm{TM}}$ autoclavable polypropylene plate for testing fermentation reaction of a particular carbohydrate namely glucose, fructose and sucrose in which a $300 \mu \mathrm{L}$ of basal media was added to each horizontal row. The plate was sealed and stored at $4^{\circ} \mathrm{C}$. Before use, it was brought to room temperature (RT) and two vertical columns were labelled with one test isolate number $(\mathrm{N})$ which pertains to native wine yeast and one column that served as control. The yeast suspension was standardized using sterile distilled water in which cell density was equivalent to the prepared No. 1 McFarland $\mathrm{BaSO}_{4}$ standard. One drop of it was inoculated in the well. The microtitre plate was covered with a lid and incubated at $37^{\circ} \mathrm{C}$ for 5 days and observed daily for any change in color.

Extraction of genomic DNA. The propagated yeast culture sample from the agar slants were inoculated into separate $10 \mathrm{~mL}$ PYG broth and grown in a $30^{\circ} \mathrm{C}$ shaking incubator for $24 \mathrm{~h}$ [11].

\section{Harvesting cells from the overnight culture.} The overnight culture was poured into a $15 \mathrm{~mL}$ centrifuge tube and spun at $3600 \mathrm{rpm}$ at $4^{\circ} \mathrm{C}$ for $5 \mathrm{~min}$. The cell pellet was then resuspended in $1 \mathrm{~mL} \mathrm{H}_{2} \mathrm{O}$. These were transferred to a $1.5 \mathrm{~mL}$ microcentrifuge tube and spun at $3600 \mathrm{rpm}$ at $4^{\circ} \mathrm{C}$ for $5 \mathrm{~min}$. The supernatant obtained from this was discarded. Then the DNA sample was spun again at $13000 \mathrm{rpm}$ for $2 \mathrm{~min}$ at $4^{\circ} \mathrm{C}$. The supernatant was removed and the pellet was air dried. The dried pellet was resuspended in $50 \mu \mathrm{L}$ buffer. The DNA was stored at $-20^{\circ} \mathrm{C}$ for analysis.

DNA isolation. The DNA pellet was resuspended in $200 \mu \mathrm{L}$ Lysis Buffer and transferred to an Eppendorf tube containing $0.1 \mathrm{~mm}$ glass beads, and $400 \mu \mathrm{L}$ chloroform/ isoamyl alcohol (24:1). It was vortexed for $2 \mathrm{~min}$ using a vortex mixer. To it was added $400 \mu \mathrm{L}$ of TE buffer $\mathrm{pH} 8.0$ and mixed briefly. The solution was centrifuged for $10 \mathrm{~min}$ at maximum speed at RT. About $400 \mu \mathrm{L}$ of the aqueous layer was transferred to a new microcentrifuge tube. One $\mathrm{mL}$ ice-cold ethanol was added and mixed by inverting the tube. It was again centrifuged for 5 min at maximum speed at RT and 70\% v/v ethanol was used to wash the pellet. The pellet was later dried at RT for $\sim 5$ min. The pellet was resuspended in $500 \mu \mathrm{L}$ TE Buffer.

Purification of the crude DNA. Fifteen microliters of $2 \mathrm{mg} / \mathrm{mL}$ RNase A were added to the solution and incubated at $37^{\circ} \mathrm{C}$ for $30 \mathrm{~min}$. To this mixture, $500 \mu \mathrm{L}$ chloroform/isoamyl alcohol (24:1) was added and vortexed for $30 \mathrm{~s}$. It was then centrifuged for $5 \mathrm{~min}$ at RT. The aqueous layer was transferred to a clean $400 \mu \mathrm{L}$ microcentrifuge tube.

\section{RESUlTS AND DISCUSSION}

To it $1 \mathrm{~mL}$ ice- cold ethanol and $10 \mu \mathrm{L} 4 \mathrm{M}$ ammonium acetate were added and mixed by inverting the tube. The solution was subjected to centrifugation for $10 \mathrm{~min}$ at the maximum speed at $4^{\circ} \mathrm{C}$ and $70 \% \mathrm{v} / \mathrm{v}$ ethanol was used to wash the pellet. The pellet was dried at room temperature for $\sim 5 \mathrm{~min}$. The pellet was resuspended in $100 \mu \mathrm{L}$ TE Buffer.

Agarose gel electrophoresis (AGE). Agarose gel was prepared and heated in the oven until boiling. Promega diamond nucleic acid dye was added to the prepared agarose gel. Then this was poured into the casting tray with the well comb in place and allowed to solidify. The 
prepared agarose gel in the casting tray was placed into the gel box and was filled with TAE buffer. Then the ladder and the sample were loaded to the wells. The AGE set up was then run at $100 \mathrm{v}$ for $30 \mathrm{~min}$. The DNA fragments were then visualized using the gel doc.

PCR and DNA sequence analysis. The purified yeast gDNA sample was stored into Cryo tubes and were sent to Macrogen Korea for polymerase chain reaction (PCR) and DNA sequencing. Internal transcribed spacer (ITS) 1 served as the forward primer and ITS 4 as the reverse primer. The DNA fragments were sequenced in order to identify the particular strain of the yeast samples.

\section{Cellular morphology characterization of the} yeast isolate. Binary fission and budding are two common methods of asexual reproduction in yeasts. Usually unicellular, yeasts reproduce vegetatively by budding $[12,13]$. Bottom fermenting yeasts like the Saccharomyces pastorianus sp. and carlsbergensis strains perform unilateral budding while, most top fermenting yeast strains such as Saccharomyces cerevisiae TUM 127 replicate by multilateral budding [14]. Few yeasts, on the other hand,

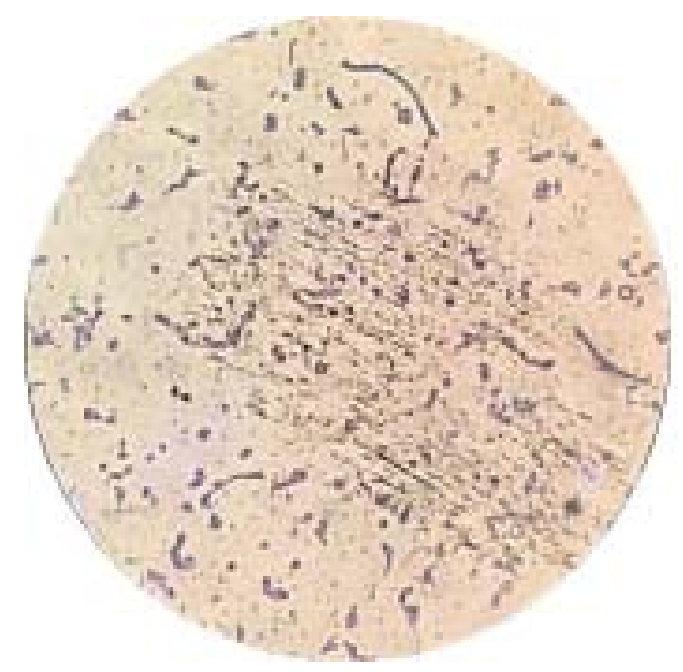

Figure 1. Photomicrograph of isolated native wine yeast under oil immersion (100× magnification). multiply by bilateral budding. Example of this are the strains of Saccharomycodes ludwigii. These budding patterns are a potential characteristic for yeast taxonomy [15].

The appearance of growth of native wine yeast on culture PYG agar medium in plates and slants was observed off-white in color. The growth was filiform and the optical property was translucent on the PYG agar slants and plates.

The photomicrograph result for native wine yeast at $100 \times$ magnification is shown in Fig. 1 . The yeast isolate reproduced by unilateral budding.

\section{Carbohydrate fermentation efficiency of the} yeast isolate. The carbohydrate fermentation test was used to discriminate bacteria from fungi [16]. Owing to the slow growth rate of fungi for which yeasts belong, prolonged incubation is recommended [17].

The carbohydrate fermentation test makes use of phenol red as a pH indicator and a general purpose fermentation medium. It is comprised of trypticase, sodium chloride, phenol red and a carbohydrate. In this study, peptone was used instead of trypticase as a source of amino acids, vitamins, minerals and other nitrogenous substances. Sodium chloride helps maintain the osmotic balance and provides the essential electrolytes for the transport into the yeast cell. Phenol red is red at neutral $\mathrm{pH}$ but turns yellow at $\mathrm{pH}<6.8-8.2$ or to magenta or hot pink at $\mathrm{pH}$ $>8.2$ [18].

The miniaturized carbohydrate fermentation test was carried out in a 9-well MicroWell ${ }^{\mathrm{TM}}$ plate. The test contained higher sugar concentration of $2 \%$. Following the procedure of Cali 2015, development of yellow color was considered as a positive result (Fig. 2).

When microorganisms ferment sugar, it produces acid and/or gas. As acid builds up, the color of phenol red changes gradually from red to yellow. 


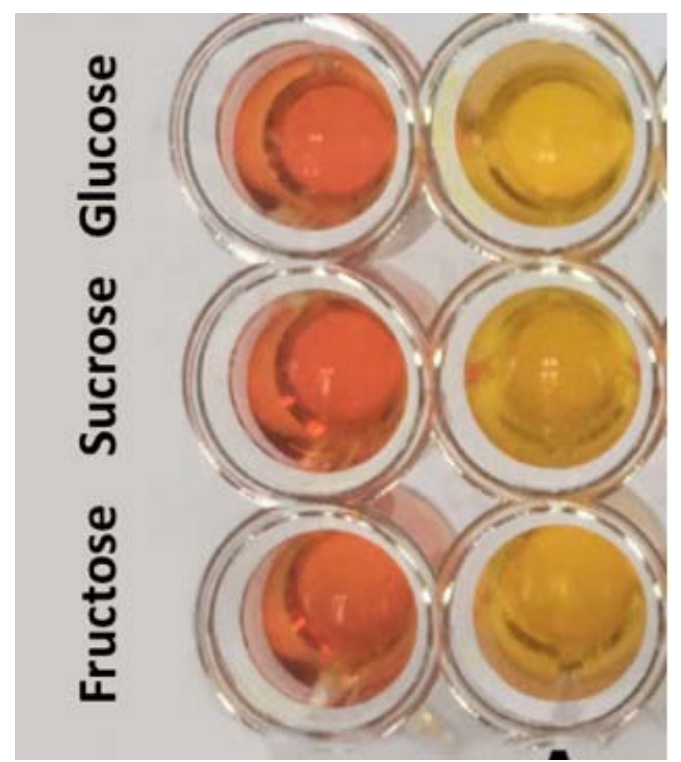

Figure 2. The test results for the miniaturized carbohydrate fermentation test after 1 week of incubation at $37^{\circ} \mathrm{C}$. The native wine yeast was inoculated in each well of the second vertical column.

Glucose undergoes fermentation when it acts as an electron donor, such as in glycolysis, and one of its metabolic products such as pyruvate act as an electron acceptor in a fermentation reaction. In fact most sugars other than glucose are said to undergo fermentation when they are either hydrolyzed into glucose or converted into glucose or both [19]. Invertase, which is enzyme present in yeasts, splits sucrose into glucose and fructose. Fructose is also possible to be converted to glucose by isomerase [20].

The results for the miniaturized carbohydrate fermentation test as shown in Fig. 2 indicated that the native yeast was positive for glucose only and a slow fermenter of other sugars.

Agarose gel electrophoresis. The AGE profile for the isolated genomic DNA samples from the native wine yeast $(\mathrm{N})$ is shown in Fig. 3. AGE is a well- known efficient and effective way to separate DNA fragments according to molecular size [21].

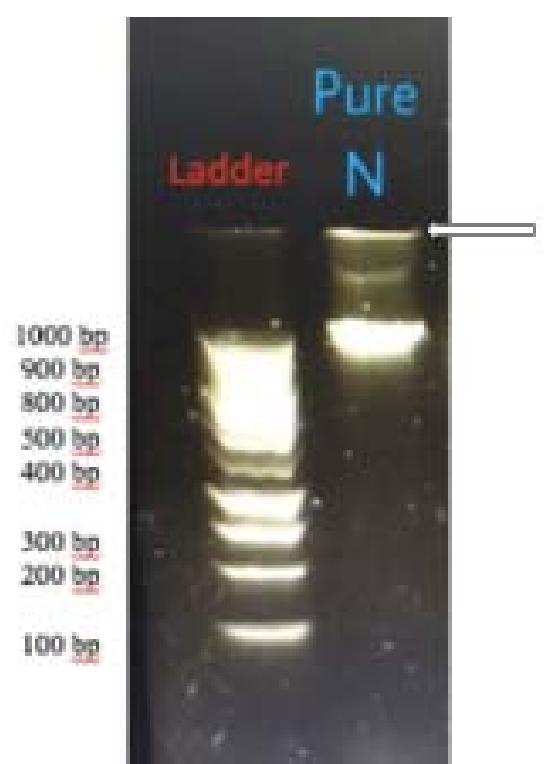

Figure 3. Age results of the purified DNA sample of native wine yeast $(\mathrm{N})$

The so called DNA ladder contains a known band sizes of DNA standards where it serves as a guide to compare and determine the approximate size of the unknown DNA band of the native wine yeast (as indicated with $\mathrm{N}$ ). A distinct band at $1000 \mathrm{bp}$ of the DNA ladder was observed as indicated by the arrow.

DNA barcoding. The native wine yeast from bubod was sent to Macrogen Korea where the sample underwent PCR amplification followed by DNA sequencing. For the PCR conditions, the predenaturation of DNA was done at $95^{\circ} \mathrm{C}$ for $5 \mathrm{~min}$, again denaturation at $95^{\circ} \mathrm{C}$ for $30 \mathrm{~s}$, followed by annealing which was done for $1 \mathrm{~min}$ at $55^{\circ} \mathrm{C}$, and extension for $1 \mathrm{~min}$ at $72^{\circ} \mathrm{C}(35$ cycles), then termination for $6 \mathrm{~min}$ at $72^{\circ} \mathrm{C}$. Lastly, cooling was done at $12^{\circ} \mathrm{C}$. Widely used primers for fungi such as ITS1 was employed as forward primer while ITS4 was employed as reverse primer. The DNA was sequenced and the chromatogram is shown in Fig. 4.

In the DNA sequencing chromatogram, the signal is extremely low and there are noise and 


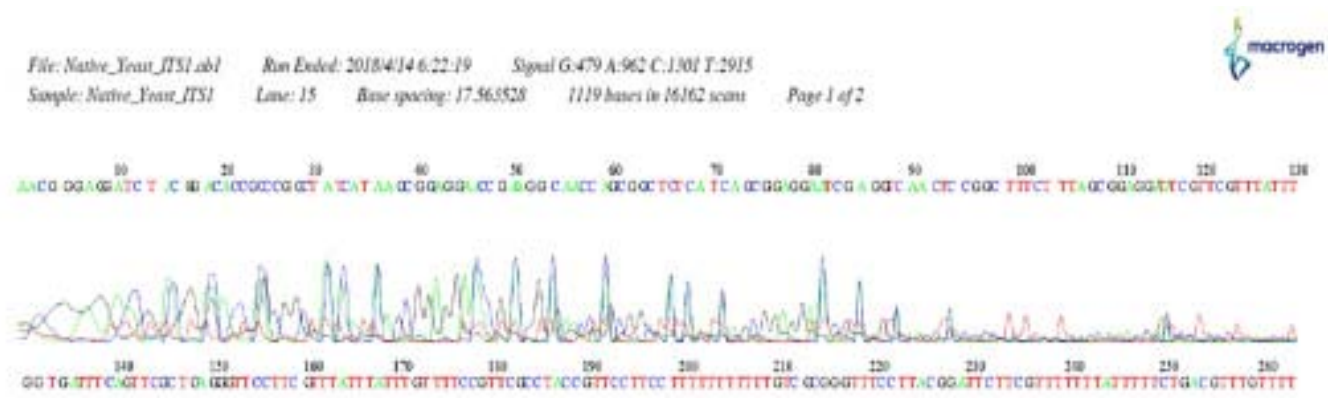

Figure 4. DNA Sequencing chromatogram of native wine yeast

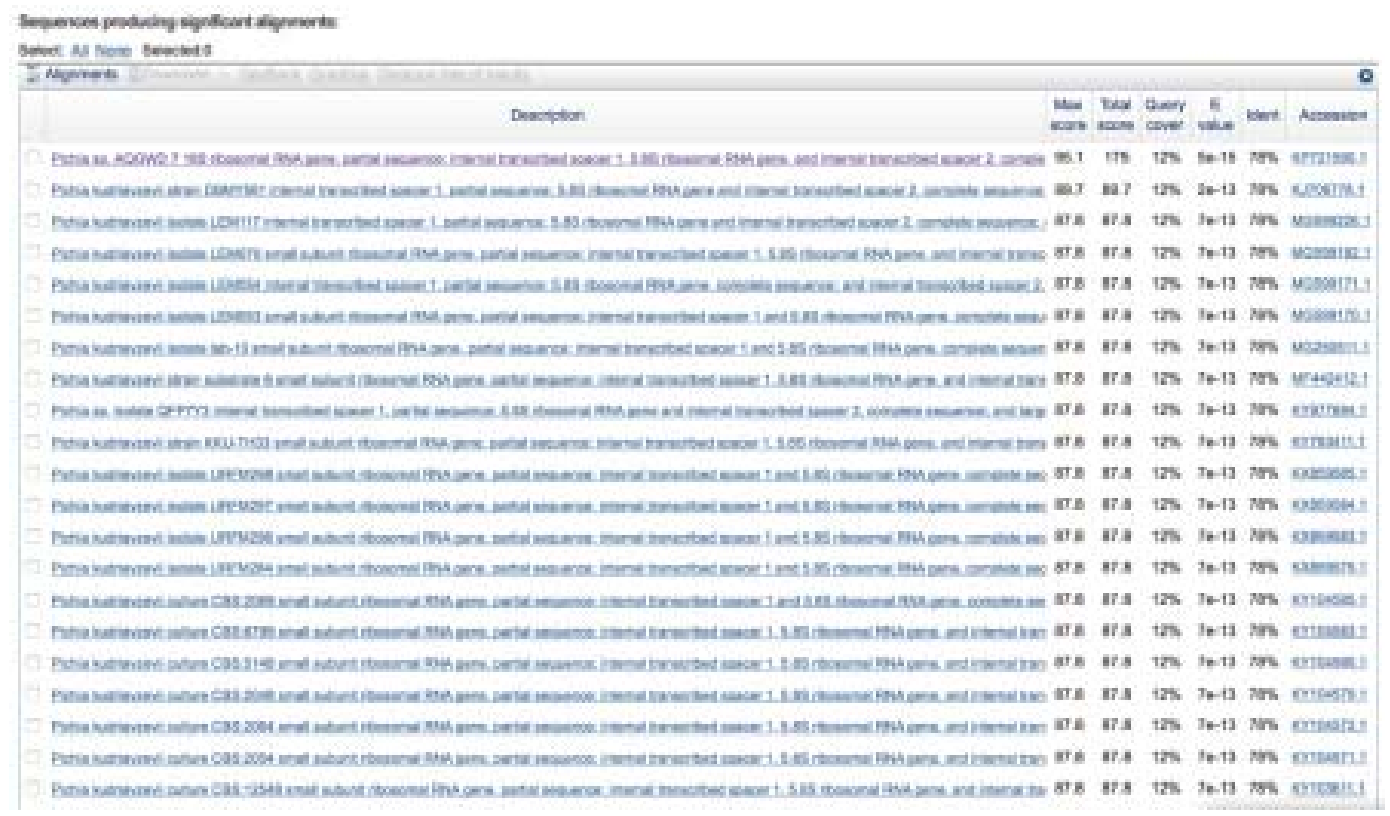

Figure 5. A BLAST table view of sequence record for a particular hit, showing each output scores and E-value.

multicolored peaks just like in $\mathrm{C}$ peaks in nucleotide 10 and nucleotide 34 though the resolution for the peaks.

This important observation may be due to the low concentration of DNA or the primer binding site is not on the sequence of sample DNA. The DNA sequence was run on nucleotide BLAST. The BLAST table view is shown in Fig. 5.

Based on the Blast result, the isolated DNA is Pichia kudriavzevii and has an identity of 78\% (146/199) and has a gap of 3\% (7/199). The E- value is a statistical parameter that provides the information if a given sequence match is purely by chance. The E-value should be near 0 and the lower it is, the more significant the match is.

According to Kurtzman, Pichia kudriavzevii cells are ovoid to elongate form, with a size of around 1.3-6 $\mu \mathrm{m} \times 3.3-14 \mu \mathrm{m}$, and occur singly or in pairs. The growth was described as butyrous and the colonies appear to be lightcream. This yeast is very abundant and widely distributed in nature often can be found in soil, on fruits, in agricultural products and foods, and 
in various natural fermentations. In fermentation, Pichia kudriavzevii strongly ferment glucose and can only utilize a limited range of sugars [22]. The findings can serve as basis for standardization of starter culture to help solve basic problem of local commercial brewers of rice wine in the Philippines to produce efficiently good quality indigenous tapuy.

\section{CONCLUSION}

The dominant native wine yeast isolated from bubod used in tapuy manufacture in the country is unicellular, reproduces by budding and works less vigorously on fermenting alcohol, which are characteristics usually of a bottom-fermentor. And that the isolated native yeast ferments glucose only within the given observation period.

DNA barcoding revealed the isolated native wine yeast species to be closely homologous to Pichia kudriavzevii with $78 \%$ identity.

\section{ACKNOWLedgements}

The authors thank John Paulin for certifying that the DNA sample isolated from the native wine yeast sent to Microgen is non-pathogenic and Mark Kevin Devanadera for assisting in the morphological characterization of the aforesaid yeast.

\section{REFERENCES}

[1] 11 Local Flavors of Wine in Philippines. FoodFindsAsia.com $\quad\left(\begin{array}{lll}2016 & 28 & \text { April }\end{array}\right)$ www.foodfindsasia.com/11-local-flavors-winephilippines/

[2] You Haven't Tasted Filipino Wine If You Haven't Savored Tapuy. Choose Philippines. www.choosephilippines.com/eat/local-flavors/ 2256/tapuy-rice-wine-traditional-filipino-ricewine/

[3] Balasubramanian MK et al. Comparative Analysis of Cytokinesis in Budding Yeast, Fission Yeast and Animal Cells. Current Biology 2004; 14(18): 806-18 DOI:10.1016/j.cub.2004.09.022
[4] Center for Food Safety and Applied Nutrition. Microorganisms \& Microbial-Derived Ingredients Used in Food (Partial List). US Food and Drug Administration Home Page, Center for Food Safety and Applied Nutrition. www.fda.gov/ Food/IngredientsPackagingLabeling/GRAS/ MicroorganismsMicrobialDerivedIngredients/ default.htm

[5] Dunn B, Levine RP, Sherlock G. Microarray karyotyping of commercial wine yeast strains reveals shared, as well as unique, genomic signatures. BMC Genomics 2005; 6(53):1-21.

[6] Philippine Rice Research Institute. Tapuy Cookbook \& Cocktails 2011.

[7] Mendoza R. Tapuy - Filipino Rice Wine: How to Make. HubPages (2015 February 27. hubpages.com/food/tapuy-filipino-rice-winehow-to-make

[8] Chinte-Sanchez P. Philippine Fermented Foods: Principles and Technology. (University of the Philippines Press, 2008).

[9] Dizon et al. Standardization of Starter Culture for Rice Wine (Tapuy) Processing. AGRIS: International Information System for the Agricultural Science and Technology. (Hillsdale, N.J.: B.L. Erlbaum Associated, 1982). 1AD, a gris.fao.org/agris-search/ search.do?recordID=PH2010000369

[10] http://Ljournal.ru/Wp-Content/Uploads/2017/03/ a-2017-023.Pdf. 2017, DOI:10.18411/a-2017023.

[11] Dymond JS. Preparation of Genomic DNA from Saccharomyces Cerevisiae. Methods in Enzymology 2013; 529:153-160. DOI:10.1016/ b978-0-12-418687-3.00012-4

[12] Objective. Asexual Reproduction in Amoeba and Yeast (Theory): Class 10: Biology: Amrita Online Lab. amrita.olabs.edu.in/ ?sub=79\&brch=16\&sim $=134 \& \mathrm{cnt}=1$

[13] Webster J, Weber R. Introduction to Fungi. (Cambridge University Press, 2007).

[14] Saccharomycetes. Saccharomycetes - An Overview ScienceDirect Topics. www.sciencedirect.com/topics/agriculturaland-biological-sciences/saccharomycetes

[15] Imanishi Y et al. Mode of Vegetative Reproduction of the Bipolar Budding Yeast Species Wickerhamomyces Pijperi and Related Strains. Microbiology 2009; 155(9):3142-48. DOI:10.1099/mic.0.029231-0

[16] Acharya T. Carbohydrate Fermentation Test: Uses, Principle, Procedure and Results. 2016, microbeonline.com/carbohydrate-fermentationtest-uses-principle-procedure-results/ 
[17] Kali A et al. A Cost-Effective Carbohydrate Fermentation Test for Yeast Using Microtitre Plate. Indian Journal of Medical Microbiology 2015; 33(2):293. DOI:10.4103/0255-0857.154884

[18] Merck. Merck Index (13 $13^{\text {th }}$ Ed). (Merck \& Co. Inc, 2001).

[19] Prescott LM et al. Microbiology (6 th $^{\text {th }}$.). (McGraw-Hill Higher Education, 2005).

[20] Fox MA, Whitesell JK. Organic Chemistry ( $3^{\text {rd }}$ Ed). (Jones and Bartlett Publishers, 2004).
[21] Kazilek. Agarose Gel Electrophoresis. (Kazilek, 2010 April 2). askabiologist.asu.edu/agarose-gelelectrophoresis

[22] Kurtzman CP, Piškur J. Taxonomy and Phylogenetic Diversity among the Yeasts. Comparative Genomics Topics in Current Genetics 2006; 29-46. DOI:10.1007/b106654 